\title{
Proposed a Framework for Depression Monitoring System by Detecting the Facial Expression using Soft Computing Algorithm
}

\author{
Sonia Sodhi, Manisha Jailia
}

\begin{abstract}
Healthcare Informatics plays a very important role for manipulating data. In the healthcare discoveries, pattern recognition is important for the prediction of depression, aggression, pain and severe disease diagnostics. In [16][5], the real innovation that has affected and organized human services is cloud computing, which empowers whenever anyplace access to the information put away in a cloud. The mobile devices are continuously observing patients that move around a networked healthcare environment. In traditional healthcare diagnostic system, we depend upon expensive tests and machineries which increase the expenditure of healthcare. It is dire need to reduce the aggregate cost of regular or usual diagnostics incorporates high cost of hospitalization. These expenses can be limited or disposed of with the assistance of remote patient monitoring gadget, a healthcare IoT product. Remote monitoring of person's health gadget includes the observing of a person from an alternate area. This dispenses the requirement for driving to clinic and to being hospitalized for less severe circumstances. This research will explore the depression monitoring system by detecting the facial expression using suitable soft computing algorithm. We may use different algorithms such as CNN and Multilayer Perceptron to get the best result. On the basis of classification it detects the class of disease. For this purpose, the primary dataset from various facial expressions of a patient will be collected, filtered and apply to classification algorithm to train the model.
\end{abstract}

Keywords: convolutional neural network, machine learning, learning (artificial intelligence).

\section{INTRODUCTION \& PROBLEM DEFINITION}

$\mathrm{H}$ ealthcare services are the major issue of our society. Till date we depend upon the analysis done by expert doctors and pathological tests. In this research, we may use soft computing algorithm to alert the patient, doctor or other paramedical services near the patient. Healthcare Informatics play very important role for manipulating the data. The Healthcare Information, learning and healthcare data continue to develop every day[8]. It's very crucial to manipulate that data and extract

Manuscript received on 31 March 2021 | Revised Manuscript received on 06 April 2021 | Manuscript Accepted on 15 May 2021 | Manuscript published on 30 May 2021.

* Correspondence Author

Sonia Sodhi*, Research Scholar, Dept. of Computer Science \& Engg., Banasthali Vidyapith University, Rajasthan, India. Email: prof_ssodhi@rediffmail.com

Manisha Jailia, Associcate Professor, Dept. of Computer Science \& Engg., Banasthali Vidyapith University, Rajasthan, India. Email: manishajailia@yahoo.co.in

(C) The Authors. Published by Lattice Science Publication (LSP). This is an open access article under the CC-BY-NC-ND license (http://creativecommons.org/licenses/by-nc-nd/4.0/) useful information from that data for building up a quality healthcare system. There is a requirement to contain the consumption on healthcare and usual monitoring, as the expenses are becoming too high. The general cost of regular or usual monitoring incorporates moving cost, hospitalization cost and examination cost. These expenses can be limited with the assistance of patient monitoring gadget [7], a healthcare IoT product. Remote monitoring of person's health gadget includes the observing of a person from an alternate area [13]. In the research problem, intend to develop a tool which will demonstrate the classification technique which is helpful in monitoring the depression of a person. We may develop a soft computing model to detect the facial expression [18] such as depression of a person[9]. In the proposed work, healthcare monitoring system by detecting facial expression such as depression of a person using soft computing technique. The model will provide early warning of depression for a person using facial expression. To develop a model, the primary dataset of various patients will be used with the consultation of physician. Once the model will be trained and will be tested on testing dataset before final implementation on real-time data. To implement the model low cost CPU enabled camera may be used such as Raspberry-Pi and a suitable language like Python may be used. In section II, state its aims and objectives. In section III, Research Methodology. In section IV, Expected Outcome of Research and In section V, Conclusion.

\section{AIMS \& OBJECTIVES OF RESEARCH WORK}

The main objectives of the proposed research work are:

- To detect depression of a person using facial expression captured by the input device.

- To optimize deep learning model that will be used to classify the depression of a person.

- To compare the results with the other proposed models to validate the working of existing model.

The aim of research work is to classify the facial expression to detect the depression of a person. To monitor the depression at initial stage of a person using an appropriate classification model is proposed deep learning [15] algorithm. It require to investigate and figure the person's depression monitoring by detecting its facial expression and for that using smart facial capturing gadget to gather information as an input for further classification.

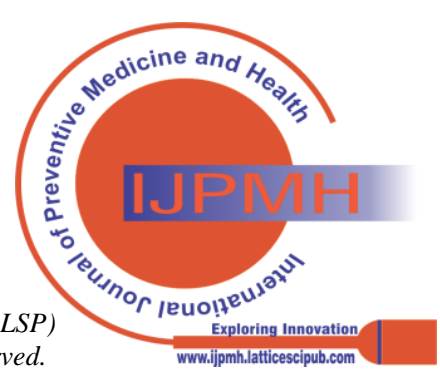




\section{RESEARCH METHODOLOGY}

It starts with the collection of training dataset captured in image form in consultation of physician at SGSS Dispensary. To capture the 2D data, we may use Raspberry-Pi with capturing device. Data may be transferred to higher end processing machine or hire cloud services of some cloud service provider. Then apply an algorithm to convert picture into quantized data, filtering the data and collect the filtered data. The data will be divided into many clusters for training purpose as well as for testing purpose using $\mathrm{k}$-fold cross validation technique. The testing of data will be done using soft computing algorithm classifier as well as other existing classifiers for comparison purpose. Once the model is ready then use it for analyzing raw data that further may be verified by the expert physician.

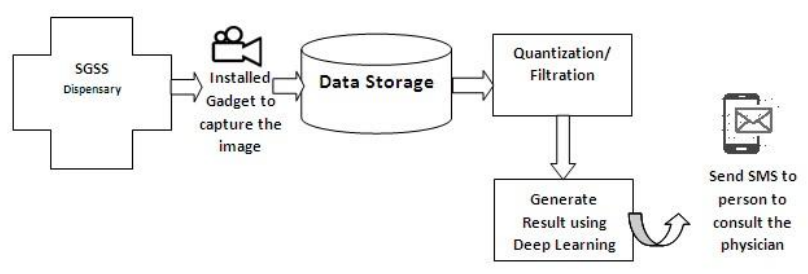

Fig. 1. Proposed Model

The algorithm to detect a patient under depression [20]: Step 1: Capture 2D primary dataset.

Step 1.1: Input N primary dataset class A, where, A data set to normal person.

Step 1. 2: Input N primary dataset class B, where, B data set to person under depression.

Step 2: Filter the 2D dataset to minimize the noise if any.

Step 3: Train the model using deep learning algorithm.

Step 4: Test the model using $M$ testing dataset where $\mathrm{M}=\mathrm{N} / 10$.

Step 5: If results are in acceptable range then go to next step elserepeatstep3aftermakingnecessarychangesinthemodel.

Step 6: Test the model with real time data and detect a person under depression.

\section{The algorithm for digital image processing [20]:}

In the representation of images one is interested in characterizing the quantity determined by each picture element. Digital processing requires that images be sampled and quantized. The rate of sampling must be sufficiently large to preserve the useful information in an image. It is determined by the image's bandwidth. Algorithm has the following steps:

\section{Step 1: Image enhancement}

The purpose of image enhancement is to emphasize those image characteristics for subsequent analysis or display of images.

\section{Step 2: Image restoration}

This refers to eliminating or mitigating documented image degradation. This includes de-blurring images that are degraded by a capture device's or its environment limitations, noise filtering, and geometric distortion correction.

Step 3: Image analysis
It is interested with producing a description of quantitative measurements from an image. This task is to read a face label point in the simplest form.

Step 4: Image data compression

The volume of visual information related data is so high that its processing will require a huge storage capacity.

\section{EXPECTED OUTCOME OF THE RESEARCH}

The expected outcome will be identifying the person under depression using the tool proposed and developed. The machine will provide early warning of depression of a patient and generate an alert which will be useful input for healthcare services of smart city for further action.

\section{CONCLUSION}

In this research, the dataset of various persons are processed and then classification algorithms are applied one by one for this dataset and results are compared to detect the depression of a person by facial expressions and implementing a tool which could help in the prediction of depression of a person using different algorithms like CNN and Multilayer Perceptron[11] to find out which one is giving better result for each attribute to predict the depression. We have to compare these algorithms and need to find out a conclusion about the best classifier on the parameters of accuracy, precision and statistics. To analyze the data to provide rapid solutions of a depression need a framework with low cost CPU enabled camera may be used such as Raspberry-Pi and a suitable language like Python. In the conclusion, this work will show that the soft computing can be a helpful tool to detect the depression of a person remotely by its facial expression which helps the person to suggest and consult the physician on time before the emergency situation occurs. In the future, we compare the results with other proposed models to validate the working of existing model and propose a tool which could help in prediction of the depression of a person by using soft computing algorithm.

\section{REFERENCES}

1. Dr. D. Venkataraman, N. S. Parameswaran, (2018), "Extraction of Facial Features for Depression Detection among Students", International Journal of Pure and Applied Mathematics, Volume 118 No. 7 2018, 455-463 ISSN: 1311-8080 (printed version); ISSN 1314-3395 (on-line version)

2. P. Raut, P. Kalbhor, H. Hirani, L. Raheja and Prof. P. Y. Pawar, (2018), "Depression Detection using BDI, Speech Recognition and Facial Recognition", International Journal for Research in Applied Science Engineering Technology (IJRASET) ISSN: 2321-9653; IC Value: 45.98; SJ Impact Factor: 6.887 Volume 6 Issue IV.

3. A. Jan, H. Meng, Y. F. Binti, A. Gaus and F. Zhang, (2018), “Artificial IntelligentSystemforAutomaticDepressionLevelAnalysisthroughVisua 1 and Vocal Expressions", IEEE Transactions on Cognitive and Developmental Systems, Vol. 10, No. 3. [CrossRef]

4. P. B. Kulkarni, M. M. Patil, (2018), "Clinical Depression Detection in Adolescent by Face", International Conference on Smart City and Emerging Technology (ICSCET). [CrossRef]

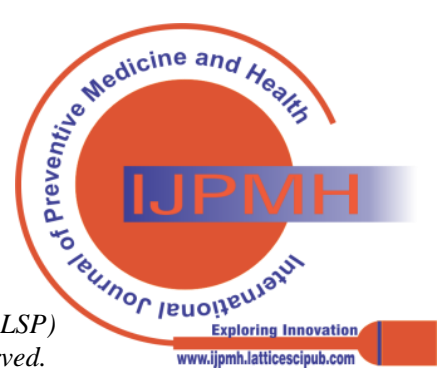


5. T. Muhammed, R. Mehmood, A. Albeshri and I. Katibi, (2018), "UbeHealth: A Personalized Ubiquitous Cloud and Edge-Enabled Networked Healthcare System for Smart Cities", Department of Computer Science, King Abdulaziz University, Jeddah 21589, Saudi Arabia 2 High Performance Computing Center, King Abdulaziz University, Jeddah 21589, Saudi Arabia, under Grant G-661-611-38.

6. S. Dham, A. Sharma and A. Dhall,

7. (2017), "Depression Scale Recognition from Audio, Visual and Text Analysis", Department of Computer Science and Engineering, Indian Institute of Technology (IIT) Ropar, India. arXiv:1709.05865v1 [cs.CV].

8. L. Fiorini, F. Cavallo, P. Dario, A. Eavis and P. C. Solly, (May 2017), "Unsupervised Machine Learning for Developing Personalised Behaviour Models Using Activity Data". [CrossRef]

9. C. Sharma and Dr. Sunanda, (2017), "Survey on Smart Healthcare: An Application of IoT".

10. S. Baker, W. Xiang and I. Atkinson, (Nov. 2017), "Internet of Things for Smart Healthcare: Technologies, Challenges, and Opportunities", IEEE. [CrossRef]

11. A. Pampouchidou, O. Simantiraki, C. - M. Vazakopoulou, C. Chatzaki, M. Pediaditis, A. Maridaki, K. Marias, P. Simos, F. Yang, F. Meriaudeau and M. Tsiknakis, (2017), "Facial Geometry and Speech Analysis for Depression Detection", 39th Annual International Conference of the IEEE Engineering in Medicine and Biology Society (EMBC). DOI: 10.1109/EMBC.2017.8037103. [CrossRef]

12. P. B. Kulkarni, M. M. Patil and V. M. Wadhai, (2017), "Review On Clinical Depression Detection Using Face And Upper Body Part", International Journal of Industrial Electronics and Electrical Engineering, ISSN: 2393-2835 Volume-5, Issue-6.

13. O. M. Alabdani, A. A. Aldahash and L. Y. AlKhalil, (2016), "A Framework for Depression Dataset to Build Automatic Diagnoses in Clinically Depressed Saudi Patients", SAI Computing Conference 2016, July 13-15,London, UK. [CrossRef]

14. J. S. Jeong, O. Han and Y. Y. You, (2016), "A Design Characteristics of Smart Healthcare System as the IoT Application", Department of Knowledge Service and Consulting, Hansung University, Seoul, 02876, Korea, Indian Journal of Science and Technology. [CrossRef

15. K. Kantharia, G. I. Prajapati, (2015), "Facial Behavior Recognition using Soft Computing Techniques: A Survey", IEEE, Fifth International Conference on Advanced Computing Communication Technologies, DOI: 10.1109/ACCT.2015.132. [CrossRef]

16. Bengio, Yoshua; LeCun, Yann; Hinton, Geoffrey (2015). ”Deep Learning”. $\quad$ Nature. 521 (7553): 436-444. Bibcode:2015Natur.521..436L. doi:10.1038/nature14539. PMID 26017442. [CrossRef]

17. A.Alkandari, M. Alnasheet andI. F. T.Alshekhly, (2012), "SmartCities Survey”, Computer Science Department, KICT, International Islamic University of Malasia Higher Institute of Telecommunication and Navigation, PAAET, Kuwait. Journal of Advanced Computer Science and Technology Research, Vol. 2 No. 2, 79-90, ISSN: 2231-8852.

18. J. F. Cohn, T. S. Kruez, I. Matthews, Y. Yang, M. H. Nguyen, M. T. Padilla, F. Zhou and F. D. L. Torre, (2009), "Detecting Depression from Facial Actions and Vocal Prosody", IEEE, 3rd International Conference on Affective Computing and Intelligent Interaction and Workshops. DOI: 10.1109/ACII.2009.5349358. [CrossRef]

19. MA Boston, (2007), "Normal vs. Abnormal Behavior: A Continuum. In: First Responder's Guide to Abnormal Psychology", Springer.

20. R. Kurt, R. Hillary, Bogner, B. Joseph, Straton,Y. Allen, Tien and J. G. Joseph, (Feb 2004), "Computer-assisted assessment of depression and function in older primary care patients", Computer Methods and Programs in Biomedicine Volume 73, Issue 2,Pages 165-171, Elsevier. https://doi.org/10.1016/S0169-2607(03)00026-9. [CrossRef]

21. G. Gupta, (2011), “Algorithm for Image Processing Using Improved Median Filter and Comparison of Mean, Median and Improved Median Filter", International Journal of Soft Comp

\section{AUTHORS PROFILE}

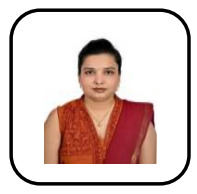

Sonia Sodhi, is a research scholar at the department of Computer Science \& Engg., Banasthali Vidyapith University, Rajasthan, India. She has done Advanced Level Course in Computer Science (ALCCS) from Institution of Electronics and Telecommunication Engineers and MCA from IGNOU. During ALCCS done six months Internship and project work done on "On The Study Of Ridge Regression Models With Crisp Inputs And Gaussian Fuzzy Outputs" under Dr. S. Balasundaram (Professor). She has cleared UGC-Net Examination in the year 2012. Worked as Adhoc and a guest faculty in the colleges of

Published By:

University of Delhi. She has presented and published a research paper in the International Conference-TelMISR-2018 entitled "Comparing performances of Support Vector Machine with conventional CPLEX, SVM ${ }^{\text {Light }}$, ASVM and LSVM" organized by Vivekananda School of Information Technology and got second prize in the International Conference.

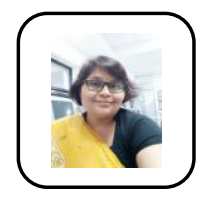

Manisha Jailia, working as Associate Professor at Banasthali Vidyapith. She has done MCA and $\mathrm{PhD}$ from Banasthali Vidyapith. Area of specialization is Data Analytics, Database Management System. Published more than 30 papers in national and International conferences and journal. 